International Journal of Algebra, Vol. 1, 2007, no. 3, 139 - 143

\title{
Ore Extensions over Pseudo-valuation Rings
}

\author{
V. K. Bhat* and Ravi Raina \\ School of Applied Physics and Mathematics, SMVD University \\ P/o Kakryal, Udhampur, J and K, India-182121 \\ *email: vijaykumarbhat2000@yahoo.com
}

\begin{abstract}
Let $\mathrm{R}$ be a commutative Noetherian Q-algebra ( $\mathrm{Q}$ is the field of rational numbers). Let $\delta$ be a derivation of $\mathrm{R}$ and $\sigma$ be an automorphism of R. Then we prove the following:

1. If $\mathrm{R}$ is a Pseudo-valuation ring, then $R[x, \delta]$ is also a Pseudo-valuation ring.

2. If $\mathrm{R}$ is a divided ring, then $R[x, \delta]$ is also a divided ring.

3. If $\mathrm{R}$ is a Pseudo-valuation ring, then $R\left[x, x^{-1}, \sigma\right]$ is also a Pseudo-valuation ring.

4. If $\mathrm{R}$ is a divided ring, then $R\left[x, x^{-1}, \sigma\right]$ is also a divided ring.
\end{abstract}

Mathemmatics Subject Classification: Primary 16-XX; Secondary 16P40, 16P50, 16U20

Keywords: Noetherian, automorphism, derivation, divided prime, pseudovaluation ring

\section{INTRODUCTION}

All rings are commutative with identity $1 \neq 0 . N(R)$ denotes the set of all nilpotent elements of $\mathrm{R}$. $\mathrm{Z}(\mathrm{R})$ denotes the centre of $\mathrm{R}$. Q denotes the field of rational numbers. We recall that as in Hedstrom [13], an integral domain $\mathrm{R}$ with quotient field $\mathrm{F}$, is called a pseudo-valuation domain (PVD) if each prime ideal $\mathrm{P}$ of $\mathrm{R}$ is strongly prime $(a b \in P, a \in F, b \in F$ implies that either $a \in P$ or $b \in P$ ). In Badawi, Anderson and Dobbs [4], the study of pseudovaluation domains was generalized to arbitrary rings. In Badawi, Anderson and Dobbs [4], a prime ideal $\mathrm{P}$ of $\mathrm{R}$ is said to be strongly prime if aP and bR are comparable (under inclusion) for all $\mathrm{a}, b \in R$. A ring $\mathrm{R}$ is said to be a pseudo-valuation ring (PVR) if each prime ideal $\mathrm{P}$ of $\mathrm{R}$ is strongly prime. We note that a PVR is quasilocal by Lemma 1(b) of Badawi, Anderson and Dobbs [4]. An integral domain is a PVR if and only if it is a PVD by Proposition (3.1) of Anderson [1], Proposition (4.2) of Anderson [2] and Proposition (3) of Badawi [5]. We recall that a prime ideal $\mathrm{P}$ of $\mathrm{R}$ is said to be divided if it is 
comparable (under inclusion) to every ideal of $\mathrm{R}$. A ring $\mathrm{R}$ is called a divided ring if every prime ideal of $\mathrm{R}$ is divided. We denote the set of prime ideals of $\mathrm{R}$ by $\operatorname{Spec}(\mathrm{R})$ and the set of strongly prime ideals of $\mathrm{R}$ by $\mathrm{S}$.Spec(R).

In Badawi [6], another generalization of PVDs is given. In Badawi [6], for a ring $\mathrm{R}$ with total quotient ring $\mathrm{Q}$ such that $\mathrm{N}(\mathrm{R})$ is a divided prime ideal of $\mathrm{R}$, let $\phi: Q \rightarrow R_{N(R)}$ such that $\phi(a / b)=a / b$ for every $a \in R$ and every $b \in R Z(R)$. Then $\phi$ is a ring homomorphism from $\mathrm{Q}$ into $R_{N(R)}$, and $\phi$ restricted to $\mathrm{R}$ is also a ring homomorphism from $\mathrm{R}$ into $R_{N(R)}$ given by $\phi(r)=r / 1$ for every $r \in R$. Denote $R_{N(R)}$ by $\mathrm{T}$. A prime ideal $\mathrm{P}$ of $\phi(R)$ is called a T-strongly prime ideal if $x y \in P, x \in T, y \in T$ implies that either $x \in P$ or $y \in P . \phi(R)$ is said to be a T-pseudo-valuation ring (T-PVR) if each prime ideal of $\phi(R)$ is T-strongly prime. A prime ideal $\mathrm{S}$ of $\mathrm{R}$ is called $\phi$-strongly prime ideal if $\phi(S)$ is a T-strongly prime ideal of $\phi(R)$. If each prime ideal of $\mathrm{R}$ is $\phi$-strongly prime, then $\mathrm{R}$ is called a $\phi$-pseudo-valuation ring $(\phi-P V R)$.

Also recall from Badawi [7], a ring $\mathrm{R}$ is called a $\phi$-chained ring $(\phi-C R)$ if $\mathrm{N}(\mathrm{R})$ is a divided prime ideal of $\mathrm{R}$ and for every $a \in T \phi(R)$, we have $a^{-1} \in \phi(R)$. In Proposition (2.6) of Badawi [8] it is shown that if $\mathrm{N}(\mathrm{R})$ is a divided prime ideal of $\mathrm{R}$ and $\mathrm{P}$ is a regular $\phi$-strongly prime ideal of $\mathrm{R}$. Then the total quotient ring $\mathrm{Q}$ of $\mathrm{R}$ is $\phi$-CR.

This article concerns the study of differential operator rings and skew Laurent polynomial rings over PVDs. Let $\mathrm{R}$ be a ring and $\delta$ be a derivation of $\mathrm{R}$. We denote the differential operator ring $R[x, \delta]$ by $\mathrm{D}(\mathrm{R})$. If I is an ideal of $\mathrm{R}$ such that $\delta(I) \subseteq I$, then we denote $I[x, \delta]$ by $\mathrm{D}(\mathrm{I})$. We would like to mention that $\mathrm{D}(\mathrm{R})$ is the usual polynomial ring with coefficients in $\mathrm{R}$ in which multiplication is subject to the relation $a x=x a+\delta(a)$ for all $a \in R$. We take any $f(x) \in R[x, \delta]$ to be of the form $f(x)=\sum x^{i} a_{i}, 0 \leq i \leq n$.

Let $\mathrm{R}$ be a ring and $\sigma$ be an automorphism of $\mathrm{R}$. We denote the skew Laurent polynomial ring $R\left[x, x^{-1}, \sigma\right]$ by $\mathrm{L}(\mathrm{R})$. We would also like to mention that in $\mathrm{L}(\mathrm{R})$ multiplication is subject to the relation $a x=x \sigma(a)$ for all $a \in R$. If I is an ideal of $\mathrm{R}$ such that $\sigma(I)=I$, then we denote $I\left[x, x^{-1}, \sigma\right]$ by $\mathrm{L}(\mathrm{I})$.

Ore-extensions including skew-polynomial rings and differential operator rings have been of interest to many authors. See $[3,9,10,11,14,15]$.

Let now $\mathrm{R}$ be a commutative Noetherian Q-algebra. Let $\delta$ be a derivation of $\mathrm{R}$ and $\sigma$ be an automorphism of $\mathrm{R}$. Then we prove the following:

1. If $\mathrm{R}$ is a Pseudo-valuation ring, then $R[x, \delta]$ is also a Pseudo-valuation ring.

2. If $\mathrm{R}$ is a divided ring, then $R[x, \delta]$ is also a divided ring.

3. If $\mathrm{R}$ is a Pseudo-valuation ring such that $\sigma(P)=P$ for all $P \in \operatorname{Spec}(R)$, then $R\left[x, x^{-1}, \sigma\right]$ is also a Pseudo-valuation ring.

4. If $\mathrm{R}$ is a divided ring such that $\sigma(P)=P$ for all $P \in \operatorname{Spec}(R)$, then $R\left[x, x^{-1}, \sigma\right]$ is also a divided ring.

These results are proved in Theorems 2.6, 2.7, 2.8 and 2.9 respectively. 


\section{Polynomial Rings}

We begin with the following known results:

Lemma 2.1. Let $R$ be a Noetherian $Q$-algebra. Let $\delta$ be a derivation of $R$. If $P$ is a prime ideal of $D(R)$, then $P \cap R$ is a prime ideal of $R$ and $\delta(P \cap R) \subseteq P \cap R$.

Proof. See Theorem (2.22) of Goodearl and Warfield [12].

Lemma 2.2. Let $R$ be a Noetherian Q-algebra. Let $\delta$ be a derivation of $R$. If $P$ is a prime ideal of $R$ such that $\delta(P) \subseteq P$, then $D(P)$ is a prime ideal of $D(R)$ and $D(P) \cap R=P$.

Proof. See Theorem (2.22) of Goodearl and Warfield [12].

Lemma 2.3. Let $R$ be a Noetherian ring. Let $\sigma$ be an automorphism of $R$. If $I$ is a prime ideal of $R$ such that $\sigma(I) \subseteq I$, then $L(I)$ is an ideal of $L(R)$ and if $J$ is an ideal of $L(R)$, then $J \cap R$ is an ideal of $R$ and $\sigma(J \cap R) \subseteq J \cap R$.

Proof. See Ex. 2ZA of Goodearl and Warfield [12].

Theorem 2.4. (Hilbert Basis Theorem): Let $R$ be a right/left Noetherian ring. Let $\alpha$ be an automorphism of $R$ and $\rho$ be an $\alpha$-derivation of $R$. Then the ore extension $O(R)=R[x, \alpha, \rho]$ is right/left Noetherian. Also $R\left[x, x^{-1}, \alpha\right]$ is Noetherian.

Proof. See Theorems (1.12) and (1.17) of Goodearl and Warfield [12].

Proposition 2.5. Let $R$ be a ring. Let $\sigma$ be an automorphism of $R$ and $\delta$ be a $\sigma$-derivation of $R$. Then:

1. For any completely prime ideal $P$ of $R$ with $\delta(P) \subseteq P$ and $\sigma(P)=P$, $P[x, \sigma, \delta]$ is a completely prime ideal of $R[x, \sigma, \delta]$.

2. For any completely prime ideal $Q$ of $R[x, \sigma, \delta], Q \cap R$ is a completely prime ideal of $R$.

Proof. See Proposition (2.4) of Bhat, Raina and Prakash [9].

Theorem 2.6. Let $R$ be a commutative Noetherian Q-algebra which is also a $P V R$. Then $D(R)$ is also a PVR.

Proof. Let $J \in \operatorname{Spec}(D(R))$. Then by Theorem (2.22) of Goodearl and Warfield [12] $J \cap R \in \operatorname{Spec}(R)$ and $\delta(J \cap R) \subseteq J \cap R$. Now $\mathrm{R}$ is a PVR, therefore $J \cap R \in S . S p e c(R)$. Now 2.5 implies that $D(J \cap R) \in S . S p e c(D(R))$. But $D(J \cap R)=J$ by Theorem (2.22) of Goodearl and Warfield [12]. Therefore $J \in S . S p e c(D(R))$. Hence $\mathrm{D}(\mathrm{R})$ is a PVR.

Theorem 2.7. Let $R$ be a divided commutative Noetherian Q-algebra. Then $D(R)$ is also divided Noetherian.

Proof. $\mathrm{D}(\mathrm{R})$ is Noetherian by 2.4. Let $J \in \operatorname{Spec}(D(R))$ and $0 \neq K$ be a proper ideal of $\mathrm{D}(\mathrm{R})$. Now by Theorem (2.22) of Goodearl and Warfield [12] 
$J \cap R \in \operatorname{Spec}(R)$ and $\delta(J \cap R) \subseteq(J \cap R)$. Also $K \cap R$ is an ideal of $\mathrm{R}$ and $\delta(K \cap R) \subseteq(K \cap R)$ by Lemma (2.18) of Goodearl and Warfield [12]. Now $\mathrm{R}$ is divided, therefore $J \cap R$ and $K \cap R$ are comparable under inclusion. Say $(J \cap R) \subseteq(K \cap R)$. Therefore $D(J \cap R) \subseteq D(K \cap R)$. Thus $J \subseteq K$. Hence $\mathrm{D}(\mathrm{R})$ is divided Noetherian.

Theorem 2.8. Let $R$ be a Noetherian ring which is also a $P V R$ and $\sigma(P)=P$ for all $P \in \operatorname{Spec}(R)$. Then $L(R)$ is also a $P V R$.

Proof. Let $J \in \operatorname{Spec}(L(R))$. Then by Ex. (2ZA) of Goodearl and Warfield [12] there exists $K \in \operatorname{Spec}(R)$ and an integer $m \geq 1$ such that $\sigma^{m}(K)=K$, and $(J \cap R)=K \cap \sigma(K) \cap \sigma^{2}(K) \ldots \sigma^{m-1}(K)$. Now by hypothesis $\sigma^{t}(K)=K$ for all integers $t \geq 1$. Therefore $(J \cap R)=K$. Now $L(K)=L(J \cap R)=J$ by (2ZA) of Goodearl and Warfield [12]. Therefore it is enough to show that $L(K) \in S . S p e c(L(R))$.

Now $K \in \operatorname{Spec}(R)$, therefore $K \in \operatorname{S.Spec}(R)$ as $\mathrm{R}$ is a PVR. Therefore by $2.5 L(K) \in S . S p e c(L(R))$. Thus $J \in S . S p e c(D(R))$. Hence $\mathrm{L}(\mathrm{R})$ is a PVR.

Theorem 2.9. Let $R$ be a divided Noetherian ring and $\sigma(P)=P$ for all $P \in \operatorname{Spec}(R)$. Then $L(R)$ is also divided.

Proof. L(R) is Noetherian by 2.4. Let $J \in \operatorname{Spec}(L(R))$. Then as in $2.8(J \cap$ $R) \in \operatorname{Spec}(R)$. Now let $\mathrm{K}$ be an ideal of $\mathrm{L}(\mathrm{R})$. Then by Ex. (2ZA) of Goodearl and Warfield [12] $K \cap R$ is an ideal of $\mathrm{R}$ and $\sigma(K \cap R)=K \cap R$. Now $\mathrm{R}$ is divided, therefore $J \cap R$ and $K \cap R$ are comparable under inclusion. Say $(J \cap R) \subseteq(K \cap R)$. Therefore $L(J \cap R) \subseteq L(K \cap R)$. Thus $J \subseteq K$. Hence $\mathrm{L}(\mathrm{R})$ is divided Noetherian.

\section{REFERENCES}

[1] D. F. Anderson, Comparability of ideals and valuation rings, Houston J. Math. 5(1979), 451-463.

[2] D. F. Anderson, When the dual of an ideal is a ring, Houston J. Math. 9(1983), 325-332.

[3] S. Annin, Associated primes over skew polynomial rings, Communications in Algebra 30 (2002) 2511-2528.

[4] A. Badawi, D. F. Anderson, and D. E. Dobbs, Pseudo-valuation rings, Lecture Notes Pure Appl. Math., Vol. 185(1997), 57-67, Marcel Dekker, New York.

[5] A. Badawi, On domains which have prime ideals that are linearly ordered, Comm. Algebra 23(1995), 4365-4373.

[6] A. Badawi, On $\phi$-pseudo-valuation rings, Lecture Notes Pure Appl. Math., Vol. 205(1999), 101-110, Marcel Dekker, New York.

[7] A. Badawi, On $\phi$-chained rings and $\phi$-valuation rings, Houston J. Math. 27(2001), 725-736.

[8] A. Badawi, On the complete integral closure of rings that admit $\phi$-strongly prime ideals, Lecture Notes Pure Appl. Math., Vol. 231(2002), 15-22, Marcel Dekker, New York.

[9] V. K. Bhat, Ravi Raina, and Om Prakash, Ore extensions over $\delta$-rigid rings, Int. J. Contem. Math. Sci. 1(12) (2006), 591-595. 
[10] V. K. Bhat, A note on Krull dimension of skew polynomial rings, Lobachevskii J. Math. 22(2006), 3-6.

[11] W. D.B lair and L.W.Small, Embedding differential and skew-polynomial rings into artinain rings, Proc. Amer, Math. Soc. 109(4) 1990, 881-886.

[12] K. R. Goodearl and R. B. Warfield Jr, An introduction to non-commutative Noetherian rings, (Cambridge Uni. Press, 1989).

[13] J. R. Hedstrom and E. G. Houston, Pseudo-valuation domains, Pacific J. Math. 4(1978), 551-567.

[14] C. Y. Hong, N. K. Kim and T. K. Kwak, Ore-extensions of baer and p.p.-rings. J.Pure and Applied Algebra 151(3) (2000), 215-226.

[15] T. K. Kwak, Prime radicals of skew-polynomial rings, Int. J. of Mathematical Sciences 2(2) (2003), 219-227.

Received: September 1, 2006 